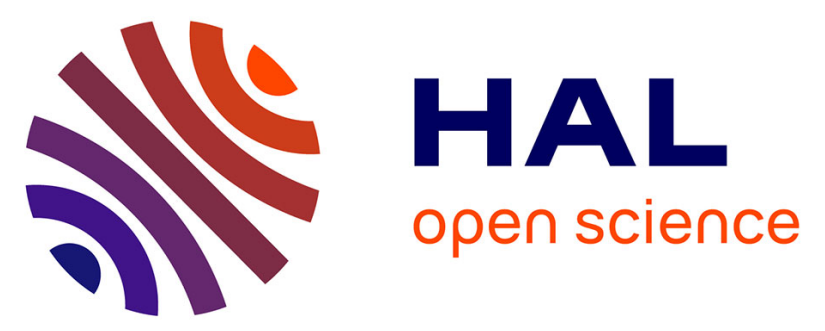

\title{
Towards Full 3D Numerical Simulation of Whirl Motions Stemming From Unilateral Contact Constraints in Aircraft Engines
}

J Paltrinieri, F Nyssen, M.-O Parent, Alain Batailly

\section{- To cite this version:}

J Paltrinieri, F Nyssen, M.-O Parent, Alain Batailly. Towards Full 3D Numerical Simulation of Whirl Motions Stemming From Unilateral Contact Constraints in Aircraft Engines. ASME Turbo Expo 2017: Turbomachinery Technical Conference and Exposition, Jun 2017, Charlotte, NC, United States. pp.GT2017-63488, 10.1115/GT2017-63488 . hal-01618311

\section{HAL Id: hal-01618311 https://hal.science/hal-01618311}

Submitted on 17 Oct 2017

HAL is a multi-disciplinary open access archive for the deposit and dissemination of scientific research documents, whether they are published or not. The documents may come from teaching and research institutions in France or abroad, or from public or private research centers.
L'archive ouverte pluridisciplinaire HAL, est destinée au dépôt et à la diffusion de documents scientifiques de niveau recherche, publiés ou non, émanant des établissements d'enseignement et de recherche français ou étrangers, des laboratoires publics ou privés. 


\title{
Towards full 3D numerical simulation of whirl motions stemming from unilateral contact constraints in aircraft engines
}

\author{
J. Paltrinieri ${ }^{1}$, F. Nyssen ${ }^{1}$, M.-O. Parent ${ }^{2}$, A. Batailly ${ }^{1}$
}

\begin{abstract}
The reduction of nominal clearances between a rotating bladed-disk and its surrounding casing yields a very significant increase of the overall engine efficiency. However, the smaller the clearances, the higher the risk of structural contacts between static and rotating components that may lead to hazardous interaction phenomena. In particular, at the fan stage of an aircraft engine, impacts between the rotating bladed-disk and the casing may generate forward or backward whirl motions induced by the precession of the shaft axis of rotation. In such specific configuration, an accurate modeling of interaction phenomena requires to account for both centrifugal and gyroscopic effects on the rotor. This contribution addresses the development of efficient reduced-order models of industrial finite element models embedding both centrifugal and gyroscopic effects. Proposed developments are validated on an academic model and are then applied on the finite element model of an aircraft engine fan stage. Results obtained with the academic model underline that the impact of gyroscopic effects on the rotor's dynamics is essentially related to the frequency split of 1-nodal diameter free-vibration modes of the first modal family. Results presented on the industrial finite element models are limited to a few case studies as a proof-of-concept.

Keywords

rotor/stator interaction; rubbing; nonlinear dynamics; unilateral contact dynamics; friction; abradable coating; fan stage
\end{abstract}

1 - Department of Mechanical Engineering, École Polytechnique de Montréal, P.O. Box 6079, Succ. Centre-Ville, Montréal, Québec, Canada H3C 3A7

2 - Safran Aircraft Engines, site de Villaroche, Moissy-Cramayel, 77550, France 


\title{
Contribution à la simulation en 3D des mouvements de précession générés par des contraintes de contact unilatérales dans les moteurs d'avion
}

\author{
J. Paltrinieri ${ }^{1}$, F. Nyssen ${ }^{1}$, M.-O. Parent ${ }^{2}$, A. Batailly ${ }^{1}$
}

\begin{abstract}
Résumé
La réduction des jeux entre les roues aubagées et les carters qui permettent des gains de performance significatifs pour le moteur favorisent aussi inévitablement l'apparition de contacts entre ces composants. Ces contacts peuvent engendrer des phénomènes d'interaction préjudiciables au bon fonctionnement du moteur. Pour la soufflante d'un moteur d'avion en particulier, des impacts répétés entre la roue aubagée en rotation et le carter peut entraîner des mouvements de précession directs ou indirects liés à un orbitage de l'axe de rotation du moteur. Dans ce cas précis, la prise en compte des effets inertiels est un pré-requis pour pouvoir envisager la prédiction numérique de ces phénomènes. Cet article porte sur le développement de modèles réduits numériques permettant la gestion de ces effets intertiels sur une large plage de vitesses de rotation. Ces développements sont tout d'abord validés sur un modèle académique et sont ensuite appliqués sur le modèle industriel éléments finis 3D d'une soufflante de moteur d'avion. Les résultats obtenus avec le modèle académique soulignent l'impact des effets gyroscopiques sur les modes de la première famille modale. Les résultats présentés sur un modèle industriel se limitent à quelques cas d'étude à titre de preuve de concept.

Mots-clés

interaction rotor/stator; frottement aube/carter; dynamique non-linéaire; dynamique du contact; frottement; revêtement abradable; soufflante

1 - Département de génie mécanique, École Polytechnique de Montréal, P.O. Box 6079, Succ. Centre-Ville, Montréal Québec, Canada H3C 3A7
\end{abstract}

2 - Safran Aircraft Engines, site de Villaroche, Moissy-Cramayel, 77550, France 


\section{INTRODUCTION}

Recent research efforts focusing on the numerical simulation of rotor/stator interactions stemming from structural contacts between blade-tips and casings in aircraft engines led to the development of an industrial computational tool [1] designed for the numerical prediction of such events. These interaction phenomena are intrisically non-linear and indeed require dedicated computational tools to overcome the current limitations of commercial softwares such as: (1) the absence of efficient strategies for the modeling of abradable coating removal [2] or, (2) the impossibility to build physically relevant reduced-order models (ROM) of rotating structures subjected to contacts [3]. The physical relevance of rotor reduced-order models is intimately related to the modeling of both centrifugal and gyroscopic effects. For most stages of an aircraft engine, accounting for centrifugal effects is of prime importance since bladed components are highly sensitive to them, particularly in fan and low-pressure stages featuring longer blades. Gyroscopic effects however typically have low to no influence on the rotor's dynamics as long as its axis of rotation is fixed. Such hypothesis is commonly accepted when investigating low- or high-pressure compressor stages. For that reason, numerical simulations of two types of rotor/stator interactions namely (1) rubbing phenomena $[4,5,6]$ and, (2) modal interaction events $[7,8]$ have been conducted without accounting for gyroscopic effects. A third type of rotor/stator interactions due to structural contacts is related to the occurence of orbital motions of an aircraft engine fan stage around its axis of rotation. These whirl motions $[9,10]$ may feature significant displacements of the center of the rotor thus considering a negligible impact of gyroscopic effects is not an acceptable assumption anymore. As a continuation of previous research work on whirl motions based on simplified 2D models [11], this contribution discusses the development of ROMs accounting for both centrifugal and gyroscopic effects over a wide angular speed range. Such models are indeed the missing step required for carrying out the first full 3D numerical simulations of whirl motions with industrial finite element models.

The simulation of whirl motions initiated by blade-tip/casing contacts is intrinsically non-linear and constitute a challenge from a numerical point of view. Such numerical investigations have been the focus of several research work carried out in collaboration with different manufacturers $[12,10,11]$. So far, and to the best of the author's knowledge, these motions have never been predicted with full 3D finite element models and there is currently no way to obtain information specific to the balde design with respect to these interactions. This level of prediction may only be reached by considering industrial finite element models which calls for the use of advanced reduction techniques in order to maintain reasonable computation times.

The first section of the article focuses on the theoretical description of gyroscopic effects modeling and their combination with ROMs of industrial finite element structures. Then, an extensive validation procedure is carried out with an emphasis on the convergence of numerical results with respect to key parameters of the simulation such as: (1) the modal reduction parameter $\eta,(2)$ the time integration step $\Delta t$ and, (3) the spatial discretization of the abradable coating deposited on the casing contact surface. The second section also features a comparison between simulations with and without gyroscopic effects on an academic model. Finally, in the last section of the article, the proposed developments are applied on the industrial finite element model of an aircraft engine fan stage.

\section{MODELING}

The gyroscopic effects affect the velocity term in the equation of motion. This term represents the conservation of the angular momentum. The angular speed is assumed to be constant in the following developments.

\section{Equation of motion}

Taking into account the centrifugal and gyroscopic effects, the equation of motion which governs the system is given by

$\mathbf{M} \ddot{\mathbf{x}}+(\mathbf{D}+\mathbf{G}(\Omega)) \dot{\mathbf{x}}+\mathbf{K}(\Omega) \mathbf{x}=\mathbf{F}$

where $\mathbf{M}, \mathbf{G}, \mathbf{K}$ and $\mathbf{D}$ denote respectively the mass, gyroscopic, stiffness and damping matrices, $\Omega$ is the angular speed, and $\mathbf{F}$ contains both the external and the contact forces. 
Denoting $\mathbf{N}$ the interpolation function matrix, the matrices in Eq. (1) are given by

$$
\begin{aligned}
\mathbf{M} & =\int_{V} \rho \mathbf{N}^{\top} \mathbf{N} \mathrm{d} V \\
\mathbf{G}(\Omega) & =\int_{V} 2 \rho \mathbf{N}^{\top} \Omega \mathbf{N} \mathrm{d} V \\
\mathbf{K}(\Omega) & =\int_{V} \varepsilon(\mathbf{x})^{\top} \mathbf{C} \varepsilon(\mathbf{x}) \mathrm{d} V \\
\mathbf{F} & =\int_{V} \mathbf{N}^{\top} \mathbf{f} \mathrm{d} V
\end{aligned}
$$

where $\rho$ is the density of the material, $\varepsilon$ the deformation tensor, $V$ the integration volume and $\mathbf{f}$ the external force.

\section{Time integration procedure}

An explicit time integration procedure is used in the paper, based on the central finitedifference method. At each time step $t$, displacements $\mathbf{x}_{t+1}$ are linearly predicted using the following scheme:

$$
\begin{aligned}
\mathbf{x}_{t+1}^{p} & =\mathbf{A} \mathbf{x}_{t}+\mathbf{B} \mathbf{x}_{t-1} \\
\mathbf{A} & =\left[\frac{\mathbf{M}}{h^{2}}+\frac{\mathbf{D}+\mathbf{G}}{2 h}\right]^{-1}\left[\frac{2 \mathbf{M}}{h^{2}}-\mathbf{K}\right] \\
\mathbf{B} & =\left[\frac{\mathbf{M}}{h^{2}}+\frac{\mathbf{D}+\mathbf{G}}{2 h}\right]^{-1}\left[\frac{\mathbf{D}+\mathbf{G}}{2 h}-\frac{\mathbf{M}}{h^{2}}\right]
\end{aligned}
$$

If a contact interaction is detected at the time step $t$, a correction is added to the predicted displacements such as:

$\mathbf{x}_{t+1}=\mathbf{x}_{t+1}^{p}+\mathbf{C} \boldsymbol{\lambda}$

where $\boldsymbol{\lambda}$ denotes the contact forces and $\mathbf{C}=\left[\frac{\mathbf{M}}{h^{2}}+\frac{\mathbf{D}+\mathbf{G}}{2 h}\right]^{-1}$. The addition of gyroscopic effects modifies the structural matrices involved in the time integration scheme, but does not affect the computational time of the procedure.

\section{Gyroscopic effects}

The gyroscopic matrix is linearly dependent on the angular speed matrix $\Omega$ which is skewsymmetric and whose three components characterize the rotation of the structure along three orthogonal axes $x, y$ and $z$, such as

$\boldsymbol{\Omega}(t)=\left(\begin{array}{ccc}0 & -\Omega_{z}(t) & \Omega_{y}(t) \\ \Omega_{z}(t) & 0 & \Omega_{x}(t) \\ -\Omega_{y}(t) & \Omega_{x}(t) & 0\end{array}\right)$

In the remainder, it is assumed that the rotor's axis of rotation is $z$. The angular speed is assumed constant and is denoted: $\Omega_{z}(t)=\Omega$. Under this assumption:

$\mathbf{K}(\dot{\Omega})=0$

and the angular speed matrix becomes

$\boldsymbol{\Omega}=\left(\begin{array}{ccc}0 & -\Omega & 0 \\ \Omega & 0 & 0 \\ 0 & 0 & 0\end{array}\right)=\Omega \underbrace{\left(\begin{array}{ccc}0 & -1 & 0 \\ 1 & 0 & 0 \\ 0 & 0 & 0\end{array}\right)}_{\mathbf{S}}$

Those approximations allow to extract the angular speed from the integral in Eq. (3), which leads to

$\mathbf{G}(\Omega)=2 \Omega\left(\mathbf{S} \otimes \mathbf{I}_{n}\right) \mathbf{M}$

where $\otimes$ and $\mathbf{I}_{n}$ respectively denote the Kronecker product and the identity matrix of dimension $n$, the number of degrees of freedom of the model. 


\section{Centrifugal effects}

The centrifugal effects affect the computation of the stiffness matrix. Since the angular speed $\Omega$ is constant, as a first approximation, one may write [13]

$\mathbf{K}(\Omega)=\mathbf{K}_{a}+\Omega^{2} \mathbf{K}_{b}+\Omega^{4} \mathbf{K}_{c}$

where the matrices $\mathbf{K}_{a}, \mathbf{K}_{b}$ and $\mathbf{K}_{c}$ are computed by computing Eq. (14) for three values of the angular speed $\Omega$, for instance, with $\Omega=0, \Omega_{\max } / 2$ and $\Omega_{\max }$. In this case, the three matrices can be expressed as $[13,14]$

$$
\begin{aligned}
& \mathbf{K}_{a}=\mathbf{K}(0) \\
& \mathbf{K}_{b}=\frac{1}{3 \Omega_{\max }^{2}}\left(16 \mathbf{K}\left(\frac{\Omega_{\max }}{2}\right)-\mathbf{K}\left(\Omega_{\max }\right)-15 \mathbf{K}(0)\right) \\
& \mathbf{K}_{c}=\frac{4}{3 \Omega_{\max }^{4}}\left(\mathbf{K}\left(\frac{\Omega_{\text {max }}}{2}\right)-4 \mathbf{K}\left(\Omega_{\max }\right)+3 \mathbf{K}(0)\right)
\end{aligned}
$$

\section{Cyclically symmetric structures}

In this study, the structures (i.e., the bladed-disk and its surrounding casing) are perfectly tuned and composed of $N$ identical sectors. As a consequence, their modes can be sorted by modal families and nodal diameters. Those properties yield symmetric mass and stiffness matrices. In addition, these matrices are block circulant in a cylindrical coordinate system, such as [15]

$$
\mathbf{Y}=\left(\begin{array}{cccccc}
\mathbf{Y}_{0} & \mathbf{Y}_{1}^{\top} & \mathbf{0} & \mathbf{0} & \ldots & \mathbf{Y}_{1} \\
\mathbf{Y}_{1} & \mathbf{Y}_{0} & \mathbf{Y}_{1}^{\top} & \mathbf{0} & \ldots & \mathbf{0} \\
\mathbf{0} & \mathbf{Y}_{1} & \mathbf{Y}_{0} & \mathbf{Y}_{1}^{\top} & \ddots & \mathbf{0} \\
\vdots & & & \ddots & & \\
\mathbf{0} & \mathbf{0} & \ldots & \mathbf{Y}_{1} & \mathbf{Y}_{0} & \mathbf{Y}_{1}^{\top} \\
\mathbf{Y}_{1}^{\top} & \mathbf{0} & \ldots & \mathbf{0} & \mathbf{Y}_{1} & \mathbf{Y}_{0}
\end{array}\right), \quad \mathbf{Y} \in\{\mathbf{K} ; \mathbf{M}\}
$$

where $\mathbf{Y}_{0}$ corresponds to one sector and $\mathbf{Y}_{1}$ to the mechanical coupling between two adjacent sectors.

In the same way, considering Eq. (13), the gyroscopic matrix is skew-symmetric and block circulant, such as

$$
\mathbf{G}(\Omega)=\left(\begin{array}{cccccc}
\mathbf{G}_{0} & \mathbf{G}_{1} & \mathbf{0} & \mathbf{0} & \ldots & \mathbf{G}_{2} \\
\mathbf{G}_{2} & \mathbf{G}_{0} & \mathbf{G}_{1} & \mathbf{0} & \ldots & \mathbf{0} \\
\mathbf{0} & \mathbf{G}_{2} & \mathbf{G}_{0} & \mathbf{G}_{1} & \ddots & \mathbf{0} \\
\vdots & & & \ddots & & \\
\mathbf{0} & \mathbf{0} & \ldots & \mathbf{G}_{2} & \mathbf{G}_{0} & \mathbf{G}_{1} \\
\mathbf{G}_{1} & \mathbf{0} & \ldots & \mathbf{0} & \mathbf{G}_{2} & \mathbf{G}_{0}
\end{array}\right)
$$

with:

$$
\begin{aligned}
& \mathbf{G}_{0}(\Omega)=2 \Omega\left(\mathbf{S} \otimes \mathbf{I}_{n}\right) \mathbf{M}_{0} \\
& \mathbf{G}_{1}(\Omega)=2 \Omega\left(\mathbf{S} \otimes \mathbf{I}_{n}\right) \mathbf{M}_{1} \\
& \mathbf{G}_{2}(\Omega)=2 \Omega\left(\mathbf{S} \otimes \mathbf{I}_{n}\right) \mathbf{M}_{1}^{\top}
\end{aligned}
$$

where $n$ represents the number of degrees of freedom of an elementary sector. Additionally, the projection onto the Fourier space (by means of the Fourier matrix $\mathcal{F}$ ) leads to mass and stiffness matrices $\tilde{\mathbf{M}}$ and $\tilde{\mathbf{K}}$, organized by spatial harmonic $n_{\mathrm{d}}$, which are symmetric and block diagonal

$$
\begin{aligned}
\tilde{\mathbf{Y}} & =\mathcal{F}^{\boldsymbol{\top}} \mathbf{Y} \mathcal{F} \\
& =\operatorname{diag}\left(\hat{\mathbf{Y}}^{(0)}, \hat{\mathbf{Y}}^{(1)}, \hat{\mathbf{Y}}^{(2)}, \ldots, \hat{\mathbf{Y}}^{(\lfloor N / 2\rfloor)}\right), \mathbf{Y} \in\{\mathbf{K} ; \mathbf{M}\}
\end{aligned}
$$


in which:

$$
\begin{aligned}
& \hat{\mathbf{Y}}^{\left(n_{\mathrm{d}}\right)}= \\
& \left(\begin{array}{cc}
\mathbf{Y}_{0}+\left(\mathbf{Y}_{1}+\mathbf{Y}_{1}^{\boldsymbol{\top}}\right) \cos \left(n_{\mathrm{d}} \alpha\right) & \left(\mathbf{Y}_{1}-\mathbf{Y}_{1}^{\boldsymbol{\top}}\right) \sin \left(n_{\mathrm{d}} \alpha\right) \\
\left(\mathbf{Y}_{1}^{\boldsymbol{\top}}-\mathbf{Y}_{1}\right) \sin \left(n_{\mathrm{d}} \alpha\right) & \mathbf{Y}_{0}+\left(\mathbf{Y}_{1}+\mathbf{Y}_{1}^{\boldsymbol{\top}}\right) \cos \left(n_{\mathrm{d}} \alpha\right)
\end{array}\right)
\end{aligned}
$$

for $n_{\mathrm{d}}=0, \ldots,\left\lfloor\frac{N}{2}\right\rfloor$. The $\lfloor\bullet\rfloor$ symbol means to the integer part.

The same projection onto the Fourier space can be done for the gyroscopic matrix, leading to a skew-symmetric and block diagonal matrix

$$
\begin{aligned}
\tilde{\mathbf{G}} & =\mathcal{F}^{\top} \mathbf{G} \mathcal{F} \\
& =\operatorname{diag}\left(\hat{\mathbf{G}}^{(0)}, \hat{\mathbf{G}}^{(1)}, \hat{\mathbf{G}}^{(2)}, \ldots, \hat{\mathbf{G}}^{(\lfloor N / 2\rfloor)}\right)
\end{aligned}
$$

with $\hat{\mathbf{G}}^{\left(n_{\mathrm{d}}\right)}=2 \Omega\left(\mathbf{S} \otimes \mathbf{I}_{n}\right) \mathbf{M}^{\left(n_{\mathrm{d}}\right)}$.

\section{Modal reduction}

A modal reduction method is used to reduce the dimension of the model. The Craig-Bampton method [16] is considered here because it advantageously retains physical degrees of freedom that are needed for accurate contact management.

Using the change of variable

$$
\mathbf{x}=\mathbf{\Phi q}
$$

where $\boldsymbol{\Phi}$ is the reduction matrix of the Craig-Bampton method [16] in which $\eta$ modes are retained and $\mathbf{q}$ is the reduced coordinates, the equation of motion in the modal space becomes

$$
\begin{aligned}
\boldsymbol{\Phi}^{\top} \tilde{\mathbf{M}} \boldsymbol{\Phi} \ddot{\mathbf{q}} & +\left(\boldsymbol{\Phi}^{\top} \tilde{\mathbf{D}} \boldsymbol{\Phi}+\boldsymbol{\Phi}^{\top} \tilde{\mathbf{G}} \boldsymbol{\Phi}\right) \dot{\mathbf{q}} \\
& +\boldsymbol{\Phi}^{\top} \tilde{\mathbf{K}}(\Omega) \boldsymbol{\Phi} \mathbf{q}=\boldsymbol{\Phi}^{\top} \mathbf{F}
\end{aligned}
$$

Denoting the reduced matrices with the index $r\left(\mathbf{A}_{\mathbf{r}}=\boldsymbol{\Phi}^{\boldsymbol{\top}} \tilde{\mathbf{A}} \mathbf{\Phi}\right)$, Eq. (27) now reads

$\mathbf{M}_{r} \ddot{\mathbf{q}}+\underbrace{\left(\mathbf{D}_{r}+\Omega \mathbf{G}_{r}\right)}_{\mathbf{D}_{r}^{\prime}} \dot{\mathbf{q}}+\mathbf{K}_{r}(\Omega) \mathbf{q}=\mathbf{F}_{r}$

with $\mathbf{G}_{r}=2\left(\mathbf{S} \otimes \mathbf{I}_{n}\right) \mathbf{M}_{r}$.

One may note that the reduced gyroscopic matrix $\mathbf{G}_{r}$ is independent of the angular speed $\Omega$ and thus requires to be computed only once for a given rotating structure. The gyroscopic effects act as a modification of the damping matrix in the equation of motion, with $\mathbf{D}_{r}^{\prime}$ the modified damping matrix in Eq. (28). Also, more details with respect to the computation of $\mathbf{K}_{r}(\Omega)$ and the treatment of centrifugal effects may be found in [17].

\section{CONVENTIONS AND NOTATIONS}

In the following, all presented data are normalized for the sake of confidentiality. Angular speeds and frequencies are directly comparable: both quantities are expressed in Hertz prior to normalization. In terms of visual representation, the following colour code is employed for all bar graphs and plots below:

black is employed for standard results: these are obtained with converged parameters (spatial discretization of the abradable coating, time integration step, and reduction parameter),

red refers to the convergence of the results with respect to the modal reduction parameter $\eta$

green refers to the convergence of the results with respect to the spatial discretization of the abradable coating $n_{\mathrm{ab}}$,

blue indicates the graph provides insight on purely numerical parameters (convergence with respect to the time step $\Delta t$, symmetry of the simulation...). 


\section{VALIDATION ON AN ACADEMIC BLADED DISK}

For the sake of efficacy, the numerical validation of the integration of gyroscopic effects within a bladed disk ROM is carried out on a simplified academic model, see Fig. 1. Fast computations allow to conduct an in-depth validation featuring convergence analyses with respect to key parameters. This academic model has 12 blades, it is assumed perfectly cyclically symmetric with respect to its axis of rotation. In order to isolate the influence of gyroscopic effects, centrifugal effects are neglected with this academic model.

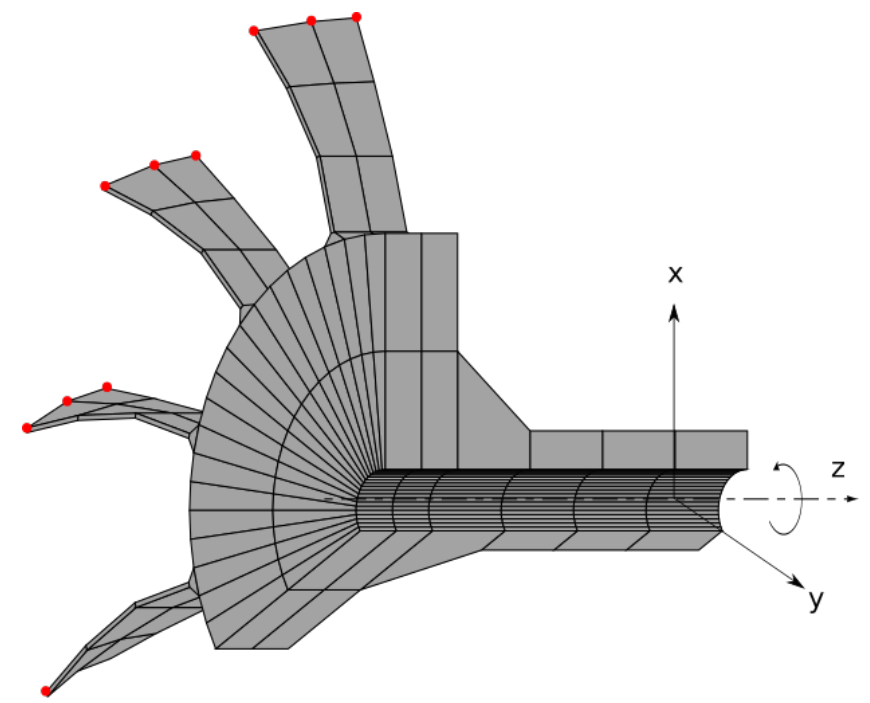

Figure 1. Academic model used for validation (only four of the twelve sectors are shown).

\section{Linear convergence}

The Campbell diagram of the academic model in the fixed intertial frame is depicted in Fig. 2 for $\Omega \in[0 ; 0.5]$. Gyroscopic effects lead to the split (see hatched areas $\square$ in Fig. 2) between the eigenfrequencies of the first harmonic of the first modal families. Since centrifugal effects are omitted, eigenfrequencies of modes for which gyroscopic terms have low to no effect appear as almost straight lines. A more thorough convergence analysis is

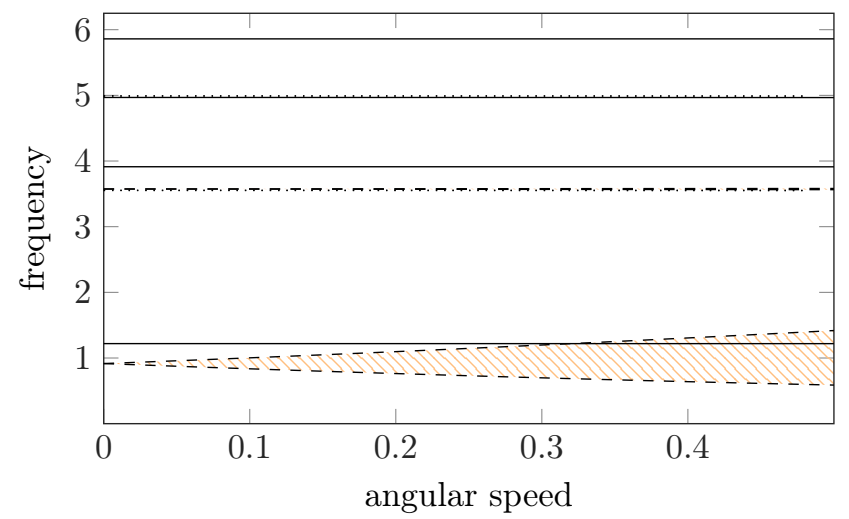

Figure 2. Campbell diagram of the academic model : harmonic $0(-)$, harmonic $1(---)$ and harmonic $2(\cdots \cdots)$.

carried out focusing on the eigenfrequencies of the first two modal families for harmonics 0 and 1 in Fig. 3. The reduction parameter $\eta$ corresponds to the number of constraint modes retained in the modal reduction basis for each of the 13 spatial harmonics ( 0 to 12) of the structure. Campbell diagrams obtained for $\eta=2,20$ and 50 are plotted in Figs. 3(a) and $3(\mathrm{~b})$ respectively for the first and the second modal family. When only two modes are retained for each harmonic, only the first modal family may be accurately represented, see Fig. 3(a). Indeed, very significant errors are observed in Fig. 3(b) for $\eta=2$. As the value of 


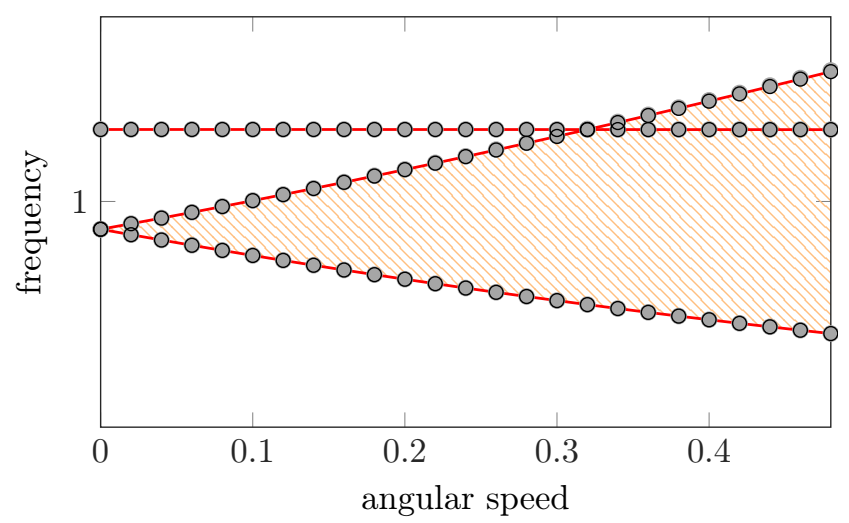

(a) modal family 1: harmonics 0 and 1

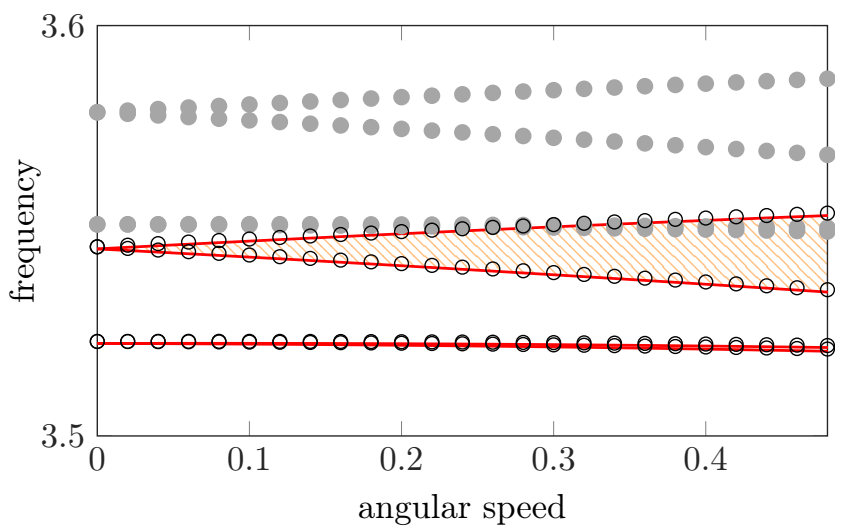

(b) modal family 2: harmonics 0 and 1

Figure 3. Campbell diagrams for different values of the modal reduction parameter: $\eta=2(\mathbf{0})$, $\eta=20(\bullet)$ and $\eta=50(-)$.

$\eta$ increases, both the first and the second modal families are very accurately represented throughout the considered angular speed range. Results for higher harmonics (2 to 6) are similar but not detailed here for the sake of brevity.

\section{Non-linear convergence}

A computational tool, developed for Safran Aircraft Engines [1], dedicated to the numerical simulation of rotor/stator interactions is here employed. As it rotates, the rotor impacts a perfectly rigid casing. Abradable coating deposited on the casing contact surface may be worn off as the blades impact the casing. Contact simulations rely on an explicit time marching procedure for which convergence must be checked with respect to key parameters including: (1) the time integration step $\Delta t,(2)$ the modal reduction parameter $\eta$ and, (3) the number of elements used for the abradable coating discretization in the circumferential direction $n_{\mathrm{ab}}$. The asymptotical convergence of the predicted results - meaning the observation of the superimposition of the results as one of these parameters is enriched - is detailed below.

First, the radial displacement $u_{\mathrm{LE}}$ of the first blade's leading edge is depicted for different time steps in Fig. 4. An all-or-nothing type of convergence is evidenced: too large time steps yield numerical divergence of the simulation soon after the first contact events (such simulations are not pictured here) while smaller time steps $\left(\Delta t \leq 5 \cdot 10^{-7} \mathrm{~s}\right)$ lead to almost perfectly superimposed results. Contrary to the time integration step $\Delta t$, the number of elements used for the abradable coating discretization has low to no effect on the computation times. For that reason, a fairly high number of elements is usually considered. Contact simulations are here performed for $n_{\mathrm{ab}}=5000,10000,15000$ and 20000 . It can be seen in Fig. 5 that for $n_{\mathrm{ab}} \geq 10000$ all the results are perfectly superimposed. The same convergence is observed with respect to the predicted wear patterns along the casing contact surface.

Finally, the asymptotical convergence of the predicted results is checked with respect to 


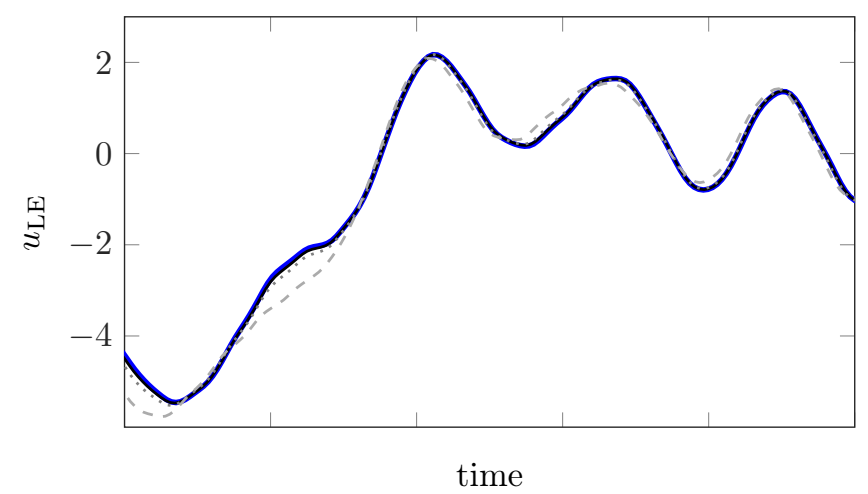

Figure 4. Predicted displacement for: $\Delta t=5 \cdot 10^{-7} \mathrm{~s}(---), \Delta t=10^{-7} \mathrm{~s}(\cdots \cdots), \Delta t=5 \cdot 10^{-8} \mathrm{~s}$ $(-)$ and $\Delta t=10^{-8} \mathrm{~s}(-) . \eta=20, n_{\mathrm{ab}}=10000$.

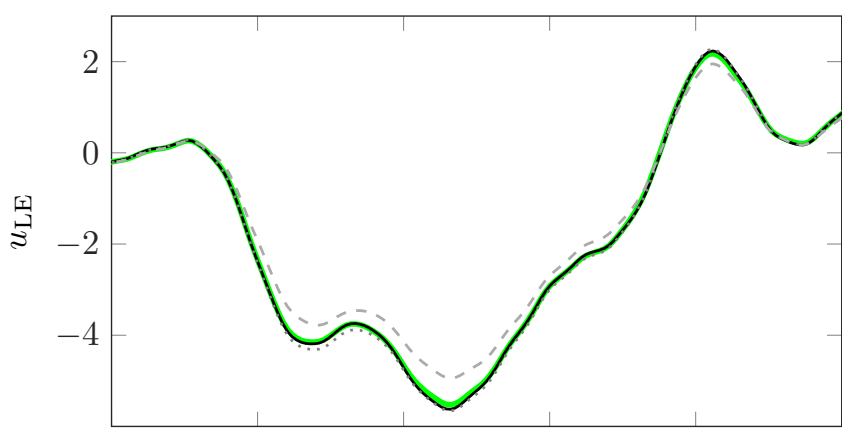

time

Figure 5. Predicted displacements for: $n_{\mathrm{ab}}=5000\left(-^{--}\right), n_{\mathrm{ab}}=10000(\cdots \cdots), n_{\mathrm{ab}}=15000(-)$ and $n_{\mathrm{ab}}=20000$ ( $) . \eta=20, \Delta t=10^{-7}$.

the modal reduction parameter $\eta$. Results depicted in Fig. 6 underline the convergence of the results as $\eta$ is increased. The participation of free-vibration modes belonging to modal families 2 and above is evidenced as $\eta=2$ yields very inaccurate displacements. For higher values of $\eta$, the good superimposition of the obtained results highlight the convergence of the numerical model.

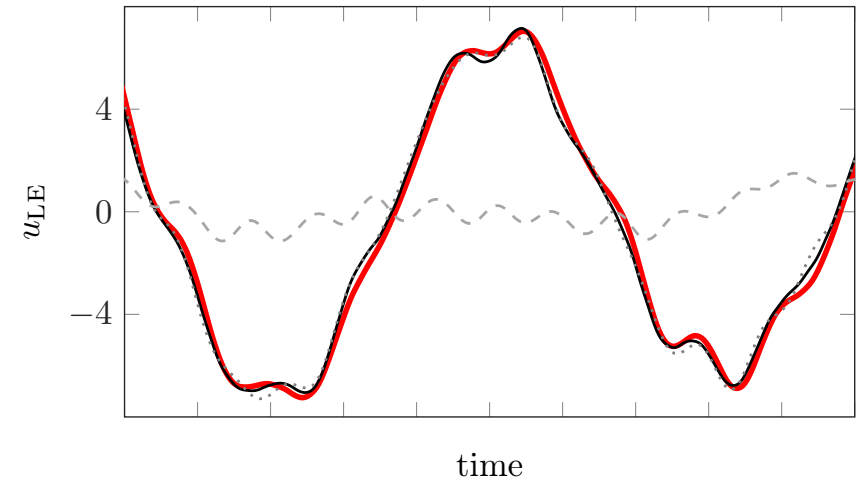

Figure 6. Predicted displacements for: $\eta=2(---), \eta=12(\cdots \cdots), \eta=20(-)$ and $\eta=50$ (-). $\Delta t=10^{-7}, n_{\mathrm{ab}}=10000$.

In order to confirm the proper numerical behaviour of the contact algorithm, a perfectly symmetric casing deformation is now considered: the casing is ovalized. The even number of blades on the rotor imposes that this configuration shall remain perfectly symmetric over time. For instance, since the academic model possesses 12 blades, the displacements predicted on blades 1 and 7 in their local frame must be identical. Displacements plotted in Fig. 7 are perfectly superimposed and thus confirm the good numerical behaviour of the employed strategy when gyroscopic effects are accounted for. 


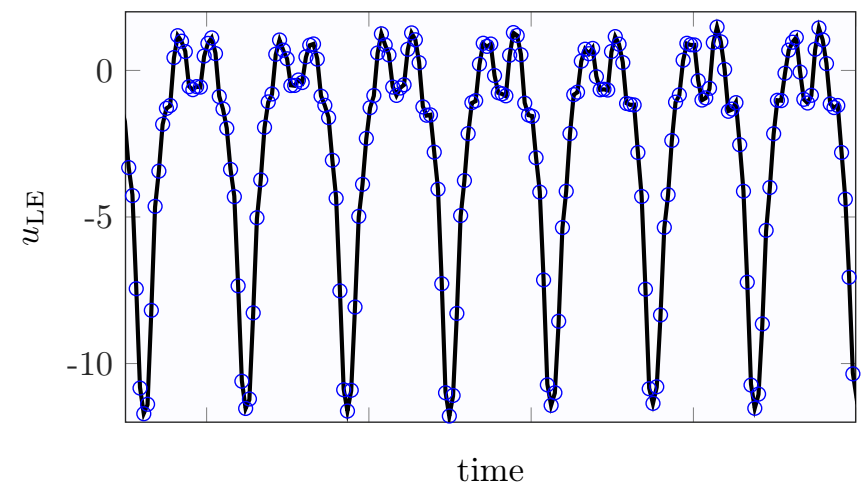

Figure 7. Axially opposed blades: radial displacements of blades $1(0)$ and 7 ( - ) leading edges.

\section{Contact simulations}

Contact simulations between the bladed disk and its surrounding casing are now carried out over a wide angular speed range $\Omega \in[0.5 ; 0.75]$. In total, a hundred simulations are performed. For each simulation, the rotor undergoes 100 revolutions and its angular speed is constant. Contact is initiated through a deformation of the rigid casing. Based on existing experimental data [18], the assumption is made that the casing is insensitive to contact, it is thus modeled as a perfectly rigid structure. For each simulation, once a steady state has been reached, a Fourier transform of the blade displacements is made. The spectra obtained for each simulation are put side by side in order to draw the interaction maps plotted in Figs. 8(a) and 8(b), respectively with and without gyroscopic effects. The same colour code is used for both maps: from white to black where black underlines the peaks in amplitudes of vibration.

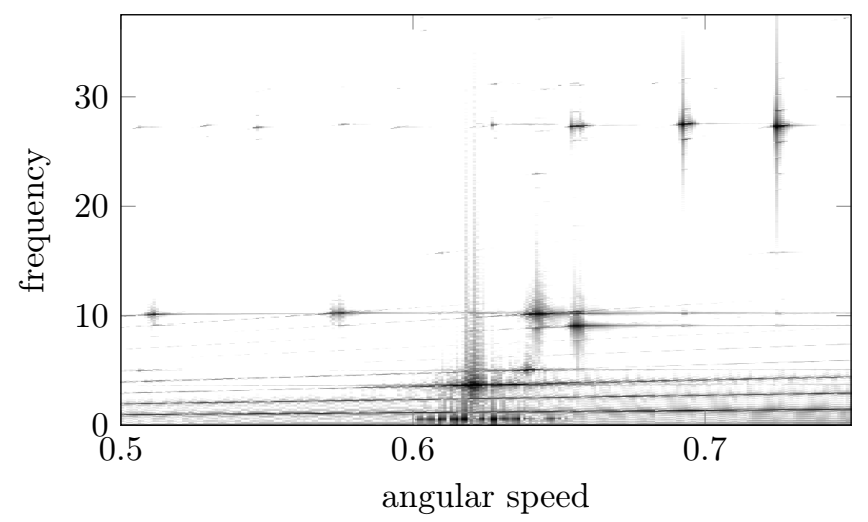

(a) with gyroscopic effects

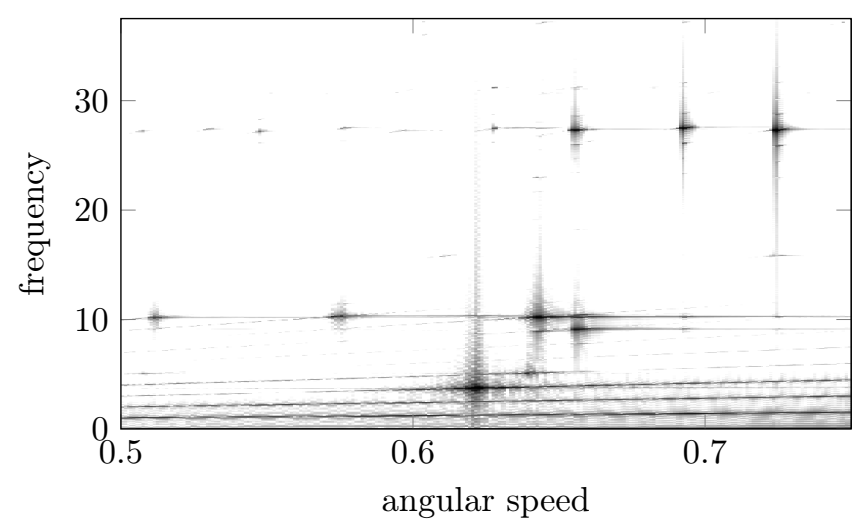

(b) without gyroscopic effects

Figure 8. Interaction maps obtained with the academic model. 
A few critical areas - areas over which peaks of amplitudes of vibration are foundare numerically predicted. Highest amplitudes of vibration are found for $\Omega \simeq 0.62$ at $f \simeq 3.5$ which suggest that the rotor vibration response is dominated by free-vibration modes belonging to the second modal family (see Figs. 2 and 3(b)). Additional peaks are evidenced at other angular speeds around $f \simeq 10$ and $f \simeq 27$ thus highlighting the key role of higher order modal families in the bladed disk's dynamics. An in-depth analysis of these interactions goes beyond the scope of the current article. Instead, the focus is made on the influence of gyroscopic effects: the subtraction of the spectra obtained with and without gyroscopic effects is plotted in Fig. 9. Non negligible differences are observed in the vicinity

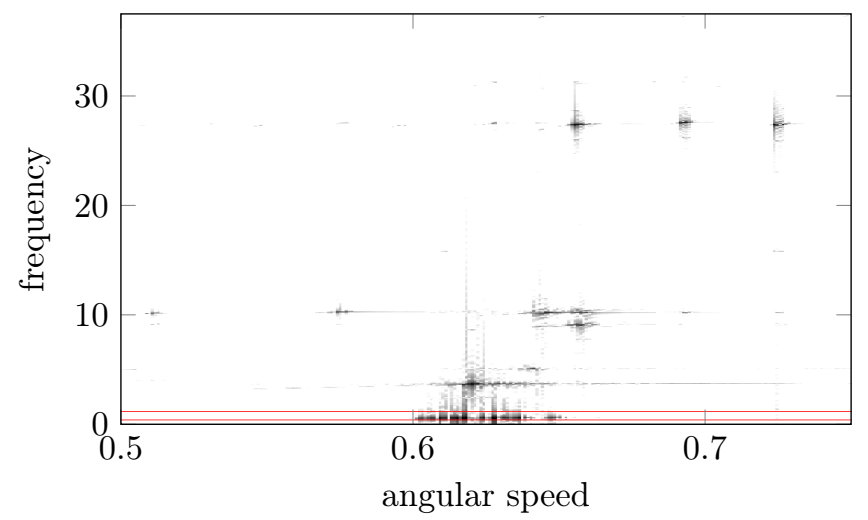

Figure 9. Subtraction between spectra plotted in Figs. 8(a) and 8(b).

of each peak of amplitudes of vibration. This is expected and simply underlines the fact that the rotor natural frequencies are affected by gyroscopic terms. This effect translates into a shift of predicted peaks both in angular speeds and frequencies hence affecting the subtraction made in Fig. 9. For $\Omega \in[0.6 ; 0.65]$, significant differences are found between the two spectra with respect to the first modal family $(f \simeq 1)$, see the red lines in Fig. 9 . The first modal family, specifically for harmonic 1, is indeed the modal family for which gyroscopic terms have the strongest effect on natural frequencies. Thus, it seems logical that differences between the spectra are located in this frequency domain. The fact that these differences are restricted to a specific angular speed range indicates that outside of this range, the first modal family has low to no effect on the rotor's steady state.

\section{INDUSTRIAL APPLICATION}

The industrial model considered in this article is that of an aircraft engine fan stage, the corresponding finite element model is partially depicted in Fig. 10. Accounting for gyroscopic effects on such stage of an aircraft engine is of particular interest as it may be subjected to orbital motions due to, for instance, residual imbalance. The system is assumed to be perfectly cyclically symmetric, it is clamped in two areas matching bearing locations, see areas in blue in Fig. 10. The finite element model contains 24 sectors and 25360 nodes per sector.

\section{Linear convergence}

A ROM of the full bladed disk is computed based on the methodology presented in [17]. Both centrifugal and gyroscopic effects are accounted for based on the methodology presented in this article. The considered angular speed range is $\Omega \in[0 ; 0.5]$. The Campbell diagram obtained with the ROM (with a modal reduction parameter $\eta=30$, computational time is a few seconds) is depicted in Fig. 11. The contribution of both centrifugal effects and gyroscopic effects are evidenced in Fig. 11: (1) centrifugal effects yield an overall increase of the fan eigenfrequencies as the angular speed increases and, (2) gyroscopic effects are responsible for the split between the eigenfrequencies of the first harmonic of the first two modal families (hatched areas $\square$ in Fig. 11). The computed ROM thus allows for an efficient extension of the reduction procedure presented in [17] to gyroscopic effects. It also yields very efficient computations for linear applications (forced responses, modal analysis or time integration). 


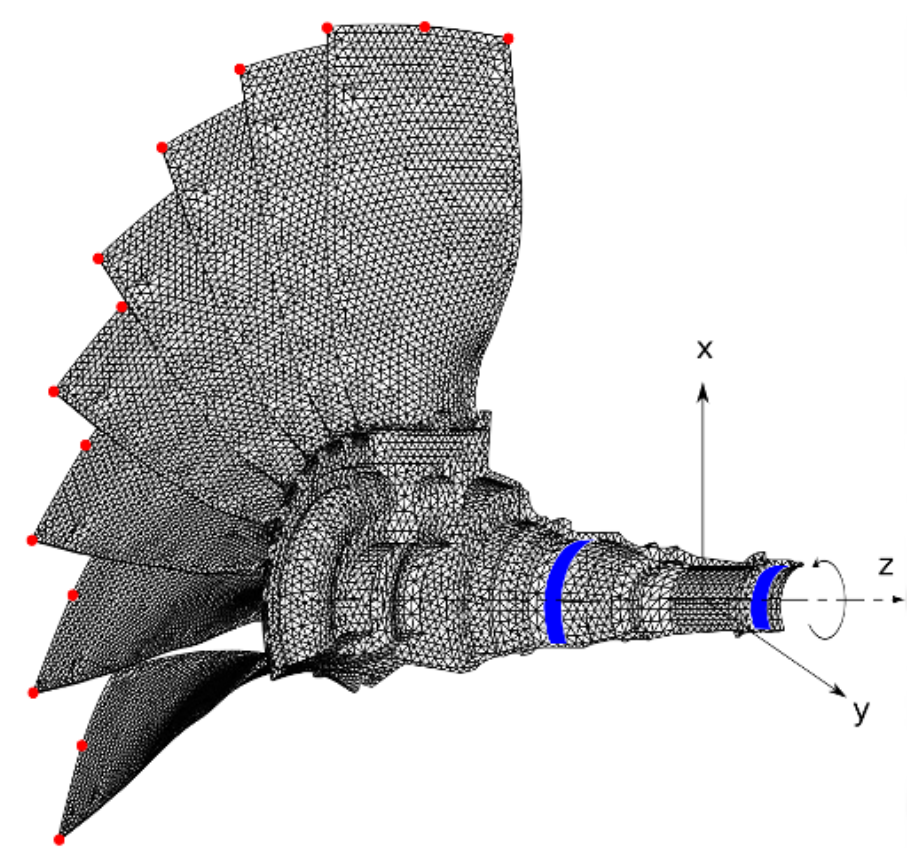

Figure 10. Partial representation of the finite element model of a civil aircraft engine fan stage. Clamped areas are marked in blue:

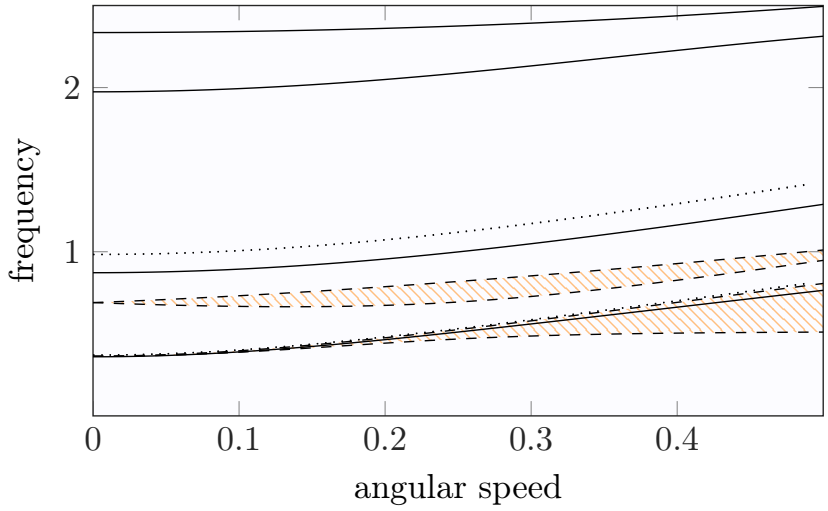

Figure 11. Campbell diagram of the investigated fan stage : harmonic $0(-)$, harmonic $1\left(--^{-}\right)$ and harmonic $2(\cdots \cdots)$.

The convergence of the eigenfrequencies of the ROM as the reduction parameter $\eta$ increases is evidenced in Figs. 12(a) and 12(b): as $\eta$ increases, the computed eigenfrequencies become perfectly superimposed throughout the angular speed range $\Omega \in[0 ; 0.5]$. One may note that the considered values of $\eta$ are much larger than in the case of the academic model, see Fig. 3. This is not related to the model itself but to the fact that centrifugal effects are here accounted for. Indeed, as detailed in [17], accounting for centrifugal effects directly within the ROM of a full bladed disk implies a significant enrichment of the modal reduction basis. Hence, $\eta$ cannot simply be interpreted as a number of contraint modes per harmonic and larger values must be considered to reach convergence all over the angular speed range of interest.

\section{Roadmap for the numerical simulation of whirl motions}

The fan stage ROM accounting for both centrifugal and gyroscopic effects may be used for the numerical prediction of sophisticated interaction motions. As an example, contact simulations are carried out over several revolutions of the rotor, the displacement field predicted in the fan stage at a time where contacts occur on two of its blades is depicted in Fig. 13. The chosen color code underlines that the shaft axis of rotation undergoes significant displacements. 


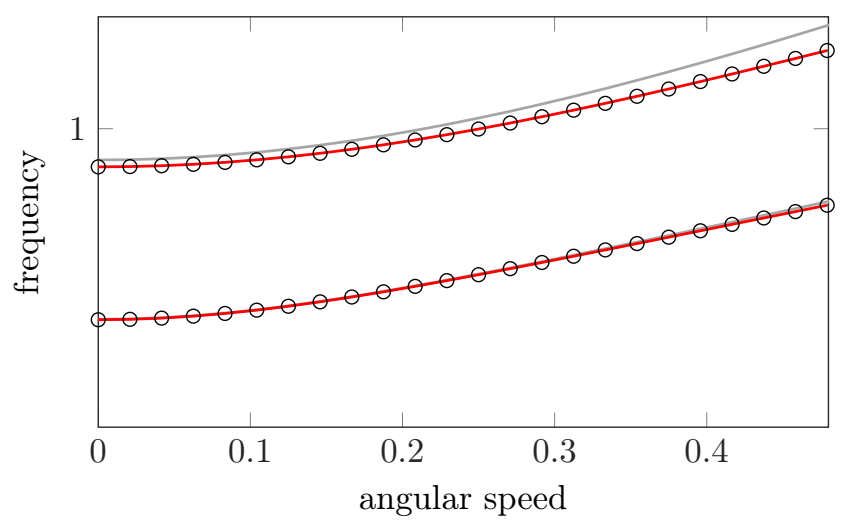

(a) modal families 1 and 2: harmonic 0

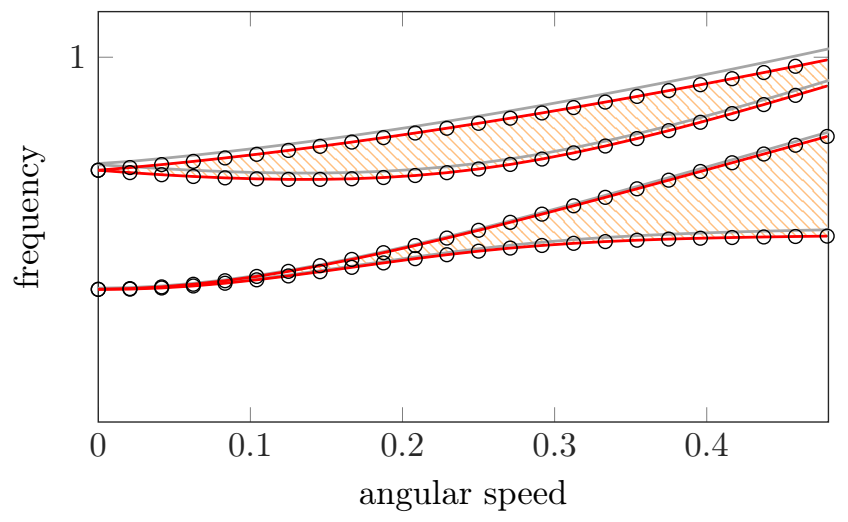

(b) modal families 1 and 2: harmonic 1

Figure 12. Campbell diagrams for different values of the modal reduction parameter: $\eta=2(-)$, $\eta=88(\longleftarrow), \eta=138$ (o) and $\eta=188(\longleftarrow)$.

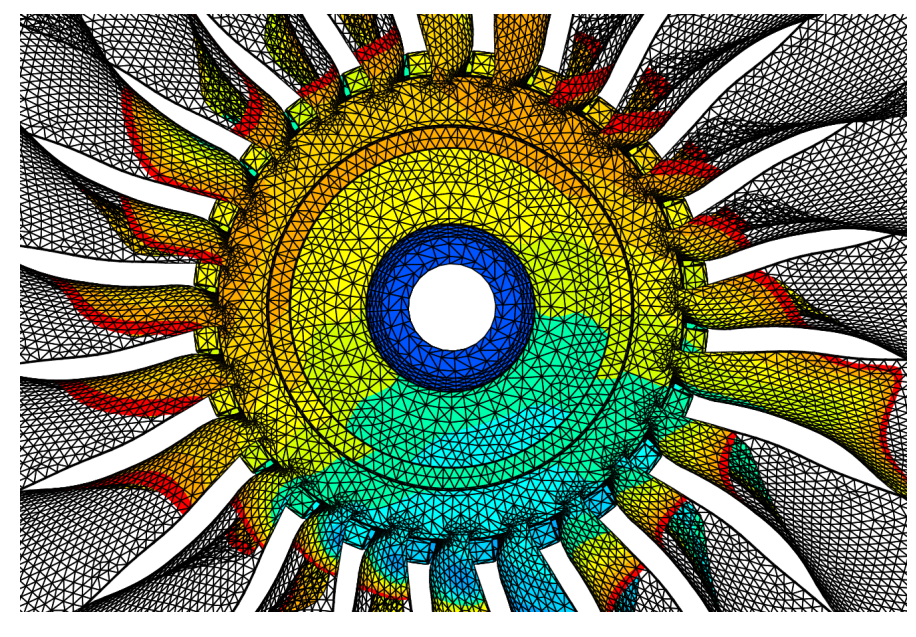

0

0.3

Figure 13. Displacement field predicted in the fan stage following contacts along blade-tips.

Providing physically realistic results requires an in-depth convergence analysis that cannot be presented here for the sake of brevity. Work is in progress to simulate whirl motions featuring significant amplitudes of vibration of the rotor around its axis of rotation. Such amplitudes may be a consequence of bearing failure - that may be easily represented numerically by removing the kinematic constraints on clamped nodes close to the bladed-disk, see Fig. 10. 


\section{CONCLUSION}

This article focuses on the modeling of centrifugal and gyroscopic effects within efficient reduced-order models. The emphasis is on the combination of gyroscopic effects - assuming a constant angular speed - with a modal reduction technique. The resulting reduced-order models advantageously feature a mixed set of reduced degrees of freedom combining both modal and physical quantities that are required for contact treatment. The possibility to embed both centrifugal and gyrocopic effects within these models opens avenues for the efficient numerical simulation of sophisticated interaction phenomena within aircraft engines as well as turbomachine components in general. This modeling strategy is applied on the fan stage of a civil aircraft engine. The accuracy of the modeling is underlined: both centrifugal and gyroscopic effects are accurately captured over a wide angular speed range at low computational cost. The presented work allows for performing the first numerical simulations of whirl motions initiated by structural contacts. The possibility to use full 3D finite element models is of prime importance for manufacturers as this paves the way for the obtention of results specific to the blade design.

\section{ACKNOWLEDGEMENT}

This research was supported by the Natural Sciences and Engineering Research Council of Canada (NSERC). The authors are also grateful to the industrial partner for supporting this project, Safran Aircraft Engines.

\section{References}

[1] Millecamps, A., Batailly, A., Legrand, M., and Garcin, F., 2015. "Snecma's viewpoint on the numerical and experimental simulation of blade-tip/casing unilateral contacts". In Proceedings of the ASME Turbo Expo 2015 conference, GT2015-42682.

${ }^{[2]}$ Legrand, M., Batailly, A., and Pierre, C., 2011. "Numerical investigation of abradable coating removal through plastic constitutive law in aircraft engine". Journal of Computational and Nonlinear Dynamics, 7, pp. 011010-1-11.

[3] Arzina, D., 2011. Vibration analysis of compressor blade tip-rubbing. Master thesis, Cranfield University.

[4] Roques, S., Legrand, M., Cartraud, P., Stoisser, C., and Pierre, C., 2010. "Modeling of a rotor speed transient response with radial rubbing". Journal of Sound and Vibration, 329(5), pp. $527-546$.

[5] Ma, H., Wang, D., Tai, X., and Wen, B., 2015. "Vibration response analysis of blade-disk dovetail structure under blade tip rubbing condition". Journal of Vibration and Control.

[6] Emery, A., Wolak, J., Etemad, S., and Choi, S., 1983. "An experimental investigation of temperatures due to rubbing at the blade-seal interface in an aircraft compressor". Wear, 91, pp. 117-130.

[7] Schmiechen, P., 1997. "Travelling wave speed coincidence". Ph.D. thesis, Imperial College of Science, Technology and Medecine - University of London, London, England.

${ }^{[8]}$ Batailly, A., and Legrand, M., 2015. "Unilateral contact induced blade/casing vibratory interactions in impellers: analysis for flexible casings with friction and abradable coating". Journal of Sound and Vibration, 348, pp. 344-364.

[9] Sinha, S., 2004. "Dynamic characteristics of a flexible bladed-rotor with coulomb damping due to tip-rub". Journal of Sound and Vibration, 273(4-5), pp. 875 - 919.

[10] Sinha, S. K., 2013. "Rotordynamic analysis of asymmetric turbofan rotor due to fan blade-loss event with contact-impact rub loads". Journal of Sound and Vibration, 332(9), pp. 2253 - 2283.

[11] Salvat, N., Batailly, A., and Legrand, M., 2016. "Two-dimensional modeling of unilateral contact-induced shaft precessional motions in bladed-disk/casing systems". International Journal of Non-Linear Mechanics, 78, pp. 90-104. 
[12] Williams, R. J., 2011. "Simulation of blade casing interaction phenomena in gas turbines resulting from heavy tip rubs using an implicit time marching method". In Proceedings of the ASME Turbo Expo 2011 conference, GT2011-45495.

[13] Sternchüss, A., and Balmès, E., 2006. "On the reduction of quasi-cyclic disks with variable rotation speeds". Proceedings of the International Conference on Advanced Acoustics and Vibration Engineering (ISMA), pp. 3925-3939.

[14] Kaptan, F., Panning-von Scheidt, L., and Wallaschek, J., 2015. "The vibrational behavior of coupled bladed disks with variable rotational speed". In Proceedings of the $86^{\text {th }}$ Annual Meeting of the International Association of Applied Mathematics and Mechanics, pp. 255-256.

[15] Bladh, R., 2001. "Efficient predictions of the vibratory response of mistuned bladed disks by reduced order modeling". Ph.D. thesis, The University of Michigan, Ann Arbor, États-Unis.

[16] Craig, R., and Bampton, M., 1968. "Coupling of substructures for dynamic analyses". AIAA Journal, 6(7), pp. 1313-1319.

[17] Batailly, A., Meingast, M., and Legrand, M., 2015. "Unilateral contact induced blade/casing vibratory interactions in impellers: analysis for rigid casings". Journal of Sound and Vibration, 337, pp. 244-262.

[18] Millecamps, A., Brunel, J., Dufrénoy, P., Garcin, F., and Nucci, M., 2009. "Influence of thermal effects during blade-casing contact experiments". In Proceedings of the ASME 2009 IDETC \& CIE conference, ASME. 